Revistade
Economìd
Contemporâned

\title{
CONDICIONANTES DA DISPOSIÇÃO DE PARTICIPAR E ACEITAR PAGAMENTOS POR SERVIÇOS AMBIENTAIS: UM ESTUDO DE CASO NO BRASIL
}

\author{
Isaque Regis Ouverney ${ }^{a}$ \\ Ronaldo Seroa da Motta ${ }^{b}$ \\ Ramon Arigoni Ortiz \\ Paulo Sérgio Coelho ${ }^{d}$ \\ ${ }^{a}$ Mestre em Economia pelo Programa de Pós-Graduação em \\ Ciências Econômicas (PPGCE) da Universidade do Estado do Rio de Janeiro (UERJ).

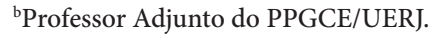 \\ 'Coordenador Técnico do Escritório de Gerenciamento de Projetos \\ (EGP-Rio/CVL) da Prefeitura do Rio de Janeiro.

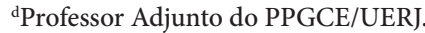

Artigo recebido em 07/04/2016 e aprovado em 07/12/2017.

RESUMO: Este estudo estima a probabilidade de produtores rurais participarem de um programa de pagamentos por serviços ambientais (PSA) na bacia do rio Paraíba do Sul, Brasil, para atividades de conversão produtiva, conservação e regeneração florestal, com base numa pesquisa de campo junto aos potenciais provedores. A estratégia de identificação condiciona as funções de probabilidade a participar do programa de PSA e a aceitar o pagamento proposto a fatores econômicos e percepções com técnicas de análise fatorial e testa modelos econométricos em um e dois estágios. Os resultados apontam que a adesão a programas de PSA é influenciada não apenas por fatores relativos ao custo financeiro de oportunidade para a adoção das novas atividades, mas, principalmente, por fatores relativos ao conhecimento prévio dos provedores sobre questões relativas ao programa e suas percepções de risco e incerteza quanto ao nível de esforço necessário para a adequação as atividades oferecidas.

PALAVRAS-CHAVE: pagamentos por serviços ambientais; condicionantes econômicos; percepções de risco e incerteza, valoração contingente.

CLASSIFICAÇÃO JEL: Q20; Q51; Q58.

Correspondência para: Ronaldo Seroa da Motta

Contato: seroadamotta.ronaldo@gmail.com 


\title{
DETERMINANTS OF PARTICIPATION AND ACCEPTANCE FOR PAYMENTS FOR ENVIRONMENTAL SERVICES: A CASE STUDY IN BRAZIL
}

\begin{abstract}
This study estimates the probability of rural producers participating and accepting payments from a program of Payment for Environmental Services (PES) in the Paraíba do Sul river basin, Brazil, for productive conversion activities and conservation and regeneration of forestland, based on a survey with the potential providers. The identification strategy estimates the probability functions of participating in the PES program and of accepting the payment conditioned to economic factors and perceptions with factorial analysis techniques and econometric models in one and two stages. The results show that adherence to PSA programs is influenced not only by factors relating to the financial opportunity cost for adopting new activities, but mainly by factors relating to prior knowledge of the providers on technical issues relating to the program and their perceptions on risks and uncertainty regarding of the effort level required to adopt the program.
\end{abstract}

KEYWORDS: payments for environmental services; economic constraints; risk and uncertainty; contingent valuation. 


\section{INTRODUÇÃO'}

O uso dos recursos naturais é socialmente ineficiente quando gera custos e benefícios que não são captados pelo sistema de preços, as chamadas externalidades. Nesses casos o nível de provisão de serviços ambientais, por exemplo, não é socialmente ótimo e a literatura econômica sugere, então, a internalização destes custos e benefícios através de mecanismos que estabeleçam um preço que induza a alocação ótima dos recursos.

Esse preço pode ser estabelecido via tributos sobre o uso dos recursos ambientais, o chamado imposto pigouviano, ou ser resultante de um mercado no qual direitos de uso são transacionados, o chamado mercado coaseniano. A preferência sobre um ou outro depende da incerteza dos custos de controle de danos e dos custos de transação².

Pagamento por serviços ambientais, tal como sugerido por Wunder (2008), pode ser entendido como resultado de um mecanismo de mercado onde há transações de cunho voluntário entre provedores e usuários de serviços ambientais. Assim, os programas de PSA passaram a ter destaque por conta de sua capacidade de traduzir os benefícios gerados pelos serviços ambientais em incentivos financeiros para a sua provisão (princípio do provedor-recebedor). Sua base reside na compensação dos indivíduos que manejam sustentavelmente os recursos ambientais, ao invés de instituir somente punições para aqueles que não o fazem (princípio do poluidor-pagador).

No entanto, como discutido por Muradian et al. (2010), as experiências com os pagamentos diretos entre provedor e recebedor privados (user-financed), isto é, pagamentos diretamente financiados pelos beneficiários, tal como nos pressupostos do mercado coaseaniano, são raros. A maioria das experiências de PSA se baseia em pagamentos financiados com recursos públicos ou privados através de um orçamento previamente estabelecido. Além disso, o pagamento ofertado não resulta da interação entre demanda e oferta pelos serviços, sendo, portanto, menos eficiente.

De qualquer forma, as experiências com PSA emergem fortemente nas últimas décadas como um incentivo importante para os esforços de provisão de serviços ambientais. Essas experiências têm sido direcionadas em particular para aqueles serviços provisionados por proprietários rurais na preservação de florestas e na adoção de práticas agropecuárias sustentáveis para proteção da biodiversidade e benefícios hidrológicos (FERRARO e KISS, 2002; MÉRAL, 2012; PAGIOLA, BISHOP e LANDELL-MILLS, 2002; PAGIOLLA e PLATAIS, 2007; GÓMEZ-BAGGETHUN et al., 2010; e PESCHE et al., 2012).

1 Os autores agradecem o apoio do bolsista de Iniciação Científica Alexandre Zaire Romero (Projeto FAPERJ E-26/200.904/2015).

2 A literatura sobre o tema é abundante. Ver, por exemplo, texto seminal em Baumol e Oates (1990) ou uma síntese em língua portuguesa em Seroa da Motta (1998). 
Nos países em desenvolvimento, domina o fomento das atividades de conservação florestal, proteção de recursos hídricos e conversão produtiva com técnicas de baixo impacto ambiental. No caso dos países mais desenvolvidos, os programas se concentram na conversão tecnológica nas atividades agropecuárias visando a adoção de práticas ambientalmente menos impactantes, em particular no uso de insumos químicos e no trato do solo (ver, por exemplo, OECD, 2010 e FAO, 2011).

O Brasil apresenta também uma gama de programas de PSA com maior incidência para ações de proteção de bacias hidrográficas e manejo florestal. Entre estes, se destaca o programa Produtor de Águas, desenvolvido pela Agência Nacional de Águas (ANA) desde 2001. Seu objetivo é o de apoiar, orientar e certificar projetos de preservação florestal e reflorestamento que resultem na melhoria da qualidade, na ampliação e na regularização da oferta de água em bacias hidrográficas de importância estratégica para o país. Esse programa, inclusive, resultou em inúmeras leis estaduais, instituindo esses mecanismos com recursos públicos orçamentários.

Outra importante experiência é da Bolsa Verde, programa de retribuição por serviços de conservação ambiental, tais como a manutenção da cobertura vegetal da área onde a família beneficiada está inserida e o uso sustentável dos recursos naturais. A Bolsa Verde é associada a um programa de transferência de renda associado ao Plano Brasil sem Miséria criado em 2011. Exemplo similar é a Bolsa Floresta, implementada inicialmente no estado do Amazonas em 2007, que constitui um mecanismo de PSA voltado a moradores de Unidades de Conservação (UC), ou seja, áreas de grande valor ambiental onde predominam populações extrativistas e agricultores familiares tradicionais $^{3}$ (ver, por exemplo, YOUNG e BAKKER, 2015; LAVRATTI e TEJEIRO, 2014; MAY et al., 2012; e GUEDES e SEEHUSEN, 2011).

Estudos que analisam as experiências com PSA indicam, contudo, que existem questões econômicas e barreiras comportamentais que afetam o nível de participação, a eficácia do pagamento e, consequentemente, a eficiência dos programas de PSA. Embora a literatura citada confirme que a participação dos produtores em programas de PSA está condicionada ao custo de oportunidade (diretos e indiretos) e barreiras financeiras associadas ao esforço de provisão dos serviços, ela também indica a importância de fatores e barreiras de percepção e de comportamento e potencial para vieses de regressividade e seleção adversa.

Há, assim, evidências de que outros fatores econômicos relacionados ao câmbio de atividades produtivas, como aqueles associados ao nível de dificuldade e incerteza das 3 Desde 2007, surgiram vários projetos de lei no Congresso Nacional, como, por exemplo, o PL 792/2007,
tratando sobre o mecanismo de PSA legislando, entre outros assuntos, as formas de financiamento. 
atividades apresentadas e assimetria de informação em relação ao desenho do programa, afetam igualmente ou às vezes de forma mais significativa a decisão do provedor de participar de um programa de PSA (KLINE, ALIG e JOHNSON, 2000; SULLIVAN, AMACHER e CHAPMAN, 2005; KILGORE et al., 2008; ENGEL, PAGIOLA e WUNDER, 2008; MARTÍNEZ-CRUZ et al., 2010; MARTIN-ORTEGA, OJEA e ROUX, 2013; e GRAMMATIKOPOULOU, POUTA e SALMIOVIRTA, 2013). Isto é, o "verdadeiro custo de oportunidade" seria distinto da mera percepção de renda líquida.

Por exemplo, Zanella, Schleyer e Speelman (2014) avaliando os primeiros programas de PSA no Brasil sobre a conservação da água descobriram que o valor do pagamento não estava correlacionado com a renda, mas sim com a intensidade de trabalho domiciliar e o acesso à informação.

Há também o registro de vieses de regressividade e de seleção adversa quando os produtores de maior renda e que já estão realizando as atividades a serem subvencionadas são aqueles com maior taxa de participação e, portanto, os programas de PSA podem não estar gerando esforço adicional significativos às ações já existentes (ver, por exemplo, ALPIZAR et al., 2007; STEFANIE et al., 2008; WUNDER e PAGIOLA, 2008; VATN, 2010; GOMEZ-BAGGETHUN, 2010; e LEIMONA et al., 2015). Há registro também de que o reconhecimento de normas sociais locais sobre o programa de PSA podem afetar a disposição a participar (KERR et al., 2017).

Em suma, a renda líquida da terra per se com a provisão não revela com precisão quanto os produtores estariam dispostos a receber para alterar suas práticas de produção e uso do solo para a provisão de serviços ambientais e, portanto, há que se considerar percepções individuais e sociais dos provedores.

Adicionalmente a essa literatura de avaliação ex-post, há estudos que tentam identificar ex-ante o nível de importância desses fatores que condicionariam a participação num programa específico de PSA e, assim, tentam estimar os valores de pagamento que viabilizariam essa participação. Esses estudos procuram identificar antecipadamente os fatores que, no desenho do programa, poderiam melhorar seu desempenho. Para tal, utilizam, na sua maioria, pesquisas com técnicas de preferências declaradas que realizam pesquisas junto a uma amostra de participantes potenciais de um programa numa certa região. Nesse tipo de pesquisa apresenta-se um cenário do programa descrevendo as atividades a serem remuneradas e questionam-se os entrevistados sobre os fatores que afetariam sua participação e, em muitos casos, sobre os valores de pagamento que estariam dispostos a aceitar ${ }^{4}$

4 Métodos de preferência declarada podem ser apresentados em dois grandes grupos: valoração contingente (CVM), no qual um atributo do serviço é apresentado e para o qual o indivíduo entrevistado reage; ou ex- 
Esses estudos confirmam as evidências das avaliações anteriormente descritas das experiências já implementadas, isto é, que além do custo de oportunidade da terra, os agricultores mostram preferências heterogêneas com alta aversão a mudanças nas suas atividades de manejo e uso do solo e que preferem participar em esquemas com medidas flexíveis e menos restritivas (VANSLEMBROUCKET, 2002; RUTO e GARROD, 2009; ESPINOSA-GODED, BARREIRO-HURLÉ e RUTO, 2010; e BROCH e VEDEL, 2012). Também confirmam que a insegurança fundiária (GROSJEAN e KONTOLEON, 2009), o risco à produção de subsistência (VIJESH et al., 2013) e a falta de esquemas de crédito (CRANFORD e MOURATO, 2011) e de informação sobre serviços ambientais e experiência anterior com PSA (WEI, GUAN e ZHU, 2016) são fatores que reduzem a participação nos países em desenvolvimento.

No caso brasileiro, o estudo pioneiro de Seroa da Motta e Ortiz (2018) estima a probabilidade de produtores participarem e aceitarem pagamentos de um programa de PSA na bacia do rio Paraíba do Sul que promoveria simultaneamente atividades de conversão produtiva, conservação e regeneração florestal. Essas estimativas se utilizam dos dados de uma pesquisa de campo na qual, além das decisões de participar e de aceitar um determinado pagamento, os respondentes indicam informações sobre a rentabilidade das suas atividades e sobre as percepções de fatores de incerteza quanto a esses custos e ao programa. Os autores utilizam inúmeras variáveis de custo de oportunidade e de percepção para estimar uma função de probabilidade de aceitação a participar no programa e outra de aceitar o valor oferecido. Nos dois casos, alguns indicadores de custo de oportunidade foram significativos, evidenciando que o efeito da renda é na maioria dos casos positivo, sugerindo a presença de um possível viés de regressividade e seleção adversa. Adicionalmente, variáveis de percepção também foram significativas, em particular as que demonstraram aversão à mudança e credibilidade no programa.

Nosso estudo adota a mesma base de dados de Seroa da Motta e Ortiz (2018), mas aplica uma estratégia de identificação distinta. Primeiro, para evitar a extensa lista de variáveis, utilizamos a da análise fatorial com o intuito de reduzir o número de variáveis independentes e explicitar atitudes latentes que norteiam as decisões dos entrevistados, aumentando nossa capacidade de entendimento dos condicionantes das probabilidades estimadas. Segundo, testamos se as decisões de participar do programa e de aceitar o pagamento devem ser estimadas em conjunto, isto é, em dois estágios.

perimento de escolhas (CE ou "choice experiment"), no qual pode haver vários atributos e estes com vários níveis e o entrevistado reage a cada um em separado. $\mathrm{O}$ entendimento pelo entrevistado das questões de CE é mais difícil, embora seja a técnica mais indicada quando os atributos são relevantes e compreensíveis. 
Logo, nosso estudo deve ser considerado uma extensão do estudo de Seroa da Motta e Ortiz (2018) com uma estratégia de identificação mais apurada. Após a descrição da base de dados, as seções seguintes apresentam os modelos adotados, a base de dados e os resultados. A última seção sintetiza as principais conclusões.

\section{ESPECIFICAÇÃO DO MODELO}

Assumimos que a decisão de aceitar participar no programa A pelo $i$-ésimo proprietário rural entrevistado é influenciada por um vetor de atributos que compreende características produtivas, econômicas e de percepção do indivíduo. Uma representação linear poderia ser:

$$
A_{i}^{*}=\beta X_{i}+\varepsilon_{i}
$$

Em (1), $X_{i}$ representa o vetor de atributos que é observável e $\varepsilon_{i}$ representa o não observável.

Para analisar os condicionantes de aceitação do valor oferecido, utilizamos o método de preferência declarada conhecido como valoração contingente, no qual os entrevistados são indagados se aceitam ou não um valor oferecido.

Formalmente, nesse caso, assumimos uma função de utilidade esperada do proprietário rural $u(j, y ; s)$, onde $j=$ zero, se o proprietário mantém suas atividades existentes numa certa área da propriedade, e $j=$ um quando ele aceita prover o serviço mediante o pagamento ( $P G T O$ ) oferecido. O termo $y$ é a renda do proprietário (rural e não rural) e $s$ é um vetor de atributos que afetam a decisão de aderir ao programa. Logo $u(j, y ; s)$ é composta de um componente observável, $v(j, y ; s)$, e outro nãoobservável, $\varepsilon j$, tal que $u(j, y ; s)=v(j, y ; s)+\varepsilon j$.

Seja agora a utilidade esperada do proprietário ao aderir ao PSA em troca de um pagamento $u_{0}=u(1, y+P G T O, s)$ e para aqueles que não aderirem seja $u_{1}=u(0, y ; s)$. Logo, o proprietário decidirá aderir ao programa se:

$$
\begin{gathered}
v(1, y+P G T O ; s)+\varepsilon_{1}>v(0, y ; s)+\varepsilon_{0} \\
\text { ou } \\
v(1, y+P G T O ; s)-v(0, y ; s)>\varepsilon_{0}-\varepsilon_{1}
\end{gathered}
$$


Assumindo uma distribuição para o termo do erro, a diferença $\varepsilon_{0}-\varepsilon_{1}$ seria distribuída, por exemplo, como em modelos Logit e Probit. Ou seja, a probabilidade de o proprietário aceitar participar do PSA com o pagamento proposto seria equivalente a quando $\Delta v$ se igualasse à diferença de utilidade em (3) (HANEMANN, 1984).

A aceitação do pagamento oferecido é observável apenas para os indivíduos que aceitaram participar anteriormente no programa, podendo, assim, acarretar em um possível viés de seleção amostral. Esse tipo de viés pode ocorrer ao se estimar os condicionantes da decisão de aceitar pagamentos pela realização de atividades que gerem os serviços ambientais apenas dos indivíduos que declararam ter interesse em participar da realização de tais atividades, excluindo-se os indivíduos que declararam o contrário. Entre os indivíduos que não têm interesse em aceitar pagamentos pela realização da atividade proposta, há aqueles que não aceitaram porque a provisão do serviço ambiental proposto representa um "custo" acima do pagamento oferecido (sendo considerado um "zero genuíno"). Os que rejeitaram participar do programa por conta de insatisfações com algum aspecto do cenário do programa apresentado podem ter, contudo, uma disposição a aceitar positiva. Isto é, há a possibilidade de endogeneidade entre as funções de participação e aceitação do pagamento para cada serviço.

Ou seja, entende-se que a função de participação no programa exerce influência direta nas estimativas da função de aceitação de pagamentos, acarretando em um possível desvio nos resultados estimados pela última. Uma solução para este problema seria a aplicação do método em dois estágios de Heckman (1979). Este método propõe uma correção para o referido problema de viés de seleção causado, pois organiza o processo em dois estágios de decisão e permite o uso de diferentes variáveis explicativas em ambos os estágios.

Assim, estima-se a disposição a participar e a aceitar pagamentos com modelo Probit para cada serviço oferecido e também simultaneamente em dois estágios como forma de garantir a mitigação do viés de seleção amostral ${ }^{5}$.

Adicionalmente, no intuito de reduzir o número de variáveis independentes e explicitar atitudes latentes que norteiam as decisões dos entrevistados, como, por exemplo, em Buckley, Hynes e Mechan (2012) e Kline, Alig e Johnson (2000), adotamos a análise fatorial. Assim, o objetivo é reduzir o número de variáveis independentes e

\footnotetext{
Heckman (1979) postula que esse viés pode ser encarado como um problema de omissão de variáveis, afirmando que a estimação da variável "omitida" e sua posterior inclusão na equação de interesse resolveria a questão. A esta variável denomina-se a razão inversa de Mills (inverse Mills' ratio), correspondendo à razão entre a função de densidade de probabilidade para a função de distribuição cumulativa da distribuição da função de participação ( $1^{\circ}$ estágio). A mitigação do viés ocorre com a introdução da razão inversa de Mills na estimação da probabilidade de aceitar o pagamento oferecido ( $2^{\circ}$ estágio).
} 
melhor identificar as atitudes latentes que norteiam as decisões dos entrevistados aumentando, dessa forma, nossa capacidade de entendimento dos condicionantes das probabilidades estimadas. Dentre as várias possibilidades metodológicas da análise fatorial, foi escolhido o método de análise de fator principal (principal component factor), rotacionando-se os fatores obtidos, de forma a garantir sua melhor interpretação ${ }^{6}$.

Para testar a possibilidade de ocorrência de um possível viés de sequência ${ }^{7}$, condiciona-se a função de aceitação de pagamento para um serviço à participação em outro, bem como a variações na ordem de apresentação dos mesmos.

\section{BASE DE DADOS}

O programa proposto na região se propunha a incentivar os proprietários de terra a desenvolverem atividades que visam à proteção ambiental e ao aumento da provisão de serviços ambientais, recebendo em troca um pagamento semestral vinculado à área que dispuseram para a realização das atividades durante um período de três anos.

Buscava-se incentivar três atividades. A primeira delas foi denominada de conservação florestal e propõe-se a diminuir a degradação das áreas de floresta nativa dentro das propriedades rurais, através de ações que visam o controle de fogo, pragas, invasões, caça ilegal, atividades extrativistas ilegais, além de outras fontes de degradação. A segunda atividade incentivada constitui-se na regeneração florestal e baseia-se na tarefa de conversão de áreas de pasto degradadas em áreas de plantação de árvores nativas, sem a possibilidade de extração de renda extrativista. Por fim, a terceira atividade foi denominada de conversão produtiva e consiste na tarefa de transformar as áreas de pasto para manejo rotacional e/ou combinar a área agrícola e de pasto com a silvicultura.

Os proprietários de terra que aceitarem participar do programa devem escolher realizar pelo menos uma das atividades oferecidas, sendo franqueada a possibilidade de realizarem mais de uma.

Após responder perguntas relativas às suas características socioeconômicas e às suas percepções sobre questões relativas ao programa (como a importância que o entrevistado confere a questões ambientais e os receios existentes na participação de programas de PSA), o indivíduo é apresentado ao programa de PSA que se pretende de-

6 Dentre as possibilidades metodológicas para a execução da rotação dos fatores, optou-se pela varimax. Esta metodologia assegura que os fatores identificados não são correlacionados entre si.

7 Quando as respostas para os serviços provocam uma resposta maior quando colocada em primeiro lugar na lista devido a efeitos de substituição e de renda (BATEMAN e LANGFORD, 1997). 
senvolver na região, com o objetivo de aumentar a produção de serviços ambientais. Procura-se salientar que, uma vez que se comprometa a realizar alguma das atividades, estará sujeito a um monitoramento para verificar o cumprimento de seu compromisso, sob pena de cancelamento dos pagamentos a serem realizados.

Também se esclarece que o programa apresentado só será implementado se o orçamento que este possui for suficiente para pagar mais da metade dos proprietários que queiram aderir, enfatizando ainda que os proprietários que pedirem as quantias mais baixas como pagamento serão os primeiros a serem contemplados. Caso o orçamento do programa não seja suficiente para realizar os pagamentos para a metade dos participantes, o programa não será implantado.

Após toda a explanação sobre o funcionamento do programa e suas restrições, pergunta-se novamente se o entrevistado conseguiu compreender bem o que lhe foi apresentado. Em seguida, questiona-se se ele teria interesse em participar de um programa nesses moldes. Caso a resposta seja negativa, a entrevista se encerra.

Para o caso do indivíduo manifestar interesse em participar do programa, cada serviço lhe é apresentado individualmente e ele é questionado se aceitaria receber um determinado valor ${ }^{8}$, a cada seis meses, para a realização da atividade proposta. Caso o indivíduo afirme que aceita o montante oferecido, ele é impelido a confirmar tal resposta, respondendo se assinaria então um contrato para a prestação da atividade, caso lhe procurassem no dia seguinte.

Essa pesquisa conduzida em Seroa da Motta e Ortiz (2018) gerou uma amostra final de 1079 questionários, isto é, 1079 propriedades agrícolas divididas em sub-amostras regionais de acordo com os estados que iriam receber o programa. Do total de entrevistas realizadas, 300 se deram no estado do Rio de Janeiro, 306 em Minas Gerais e 473 em São Paulo.

Entretanto, a questão mais difícil de tratar quando se aplica métodos de preferências declaradas, muito discutida nos principais manuais (MITCHELL e CARSON, 1989; ARROW et al., 1993; SEROA DA MOTTA, 1997; BATEMAN et al., 2002; e ALBERINI e KAHN, 2006) é a do viés estratégico que pode sub ou superestimar as preferências declaradas. No caso da mensuração da disposição a aceitar, o indivíduo pode ancorar na restrição orçamentária do pagador ou também sentir que sua res-

\footnotetext{
8 Antes da formatação final do questionário, uma pesquisa piloto foi colocada em curso, totalizando 30 entrevistas onde o valor do pagamento a ser recebido pela prestação do serviço era definido pelo respondente. Com base nos valores informados neste estudo preliminar, estimou-se uma escala de dez valores de pagamentos a serem oferecidos (um por questionário) na confecção da pesquisa final. O mesmo valor era oferecido para os três serviços. A escala de valores dos pagamentos oferecidos foi a seguinte: R\$100, R\$ 200, R\$ 400, R\$ 600, R\$ 800, R\$ 1000, R\$1200, R\$ 1400, R\$ 1600 e R\$ 2000.
} 
posta poderá influenciar o pagamento final e, com isso, revelar valores mais elevados que a sua verdadeira disposição a aceitar.

Portanto, neste estudo utilizamos a técnica de corte pontual (provision point mechanism) que informa ao entrevistado a existência de um orçamento pré-fixado para o montante total do programa e a necessidade de um percentual mínimo de adesão. (THOMAS e BLAKEMORE, 2007; CRANFORD e MOURATO, 2011; LINDHJEMA e MITANI, 2012; e BUSH et al., 2013). O cenário aqui adotado foi que para viabilizar o programa, o total de pagamentos não poderia exceder certo orçamento preestabelecido (não revelado) e que a adesão deveria alcançar 50\% da área agrícola da região.

Foram implementados também dois tipos de questionários ( $X$ e $Y$ ) na construção da base de dados, onde a única diferença reside na ordem de apresentação das atividades oferecidas. O questionário do tipo $X$ oferece primeiramente ao entrevistado a opção de realizar a atividade de conservação, seguido da atividade de regeneração e da conversão produtiva, ao final. Já o questionário do tipo $Y$ oferece em primeiro lugar a atividade de conversão produtiva, seguido da regeneração e, por último, da atividade de conservação. O objetivo da implementação de dois tipos diferentes de questionários é testar o viés de sequência, no qual a ordem de apresentação das atividades afeta as decisões do indivíduo de participar e/ou aceitar pagamentos. A amostra final conta com 504 questionários do tipo $X$ e 572 do tipo $Y^{9}$.

\section{ANÁLISE FATORIAL}

Para viabilizar a construção das variáveis a serem usadas na estimação das funções de probabilidade de participação e de aceitação, as informações foram agregadas de acordo com a atividade e/ou para a propriedade como um todo.

Foi realizado o teste de Kaiser-Meyer-Olkin para verificação da adequação da $\operatorname{amostra}^{10}$, registrando uma adequação de 0,63 para o conjunto de variáveis. A definição sobre qual tipo de relação o fator explicita é feita com base nas cargas fatoriais das

9 A diferença no número de questionários não foi intencional e se deu por conta de uma significativa diferença de coleta por tipo de questionário no estado de São Paulo. Além disso, três questionários aplicados neste estado não tiveram a categorização (X ou Y) definida. Deste modo, a soma dos questionários aplicados segundo seu tipo é igual a 1076.

${ }^{10} \mathrm{O}$ critério de retenção de fatores adotado foi o critério de Kaiser, que postula que os fatores utilizados devem apresentar autovalores iguais ou superiores a uma unidade. Este teste tem como objetivo indicar a proporção da variância dos dados que é comum a todas as variáveis. Apresenta valores entre zero e 1, sendo que quanto mais próximo de 1 , mais recomendada se torna a aplicação da análise fatorial. Hair et al. (2009) sugerem 0,50 como patamar aceitável para justificar a análise fatorial. 
variáveis da amostra. Quanto maior for a carga fatorial da variável, maior sua importância para o fator em questão, sendo consideradas relevantes as variáveis que apresentam carga fatorial de, no mínimo, 0,50. Desta forma, é possível identificar as relações latentes entre as variáveis com base nas combinações existentes para cada fator ${ }^{11}$. Os resultados obtidos pela análise fatorial apontam para um conjunto de 17 fatores, que compreendem 62,55\% da variabilidade comum da amostra, conforme Anexo 1.

O primeiro fator explicitado na análise é o de Percepção Ecológica, registrando maiores cargas fatoriais em variáveis de percepção da questão ambiental, que representa as variáveis de conhecimento sobre questões ambientais globais e já ter ouvido falar sobre temas como sustentabilidade e mudança do clima. A princípio, espera-se que este fator registre uma correlação positiva com a disposição a participar e também com a disposição a aceitar pagamentos do programa, uma vez que indica que o indivíduo possui um melhor entendimento acerca dos benefícios oriundos de uma maior oferta de serviços ecossistêmicos. Caso registre um impacto negativo na probabilidade de aceitar o pagamento oferecido, pode ser um indicativo da ocorrência de um possível viés estratégico.

O fator Área Utilizada, por sua vez, tem suas maiores cargas fatoriais relacionadas com variáveis produtivas, que expressam a dimensão dos lotes das propriedades localizadas na região onde a pesquisa foi realizada. Espera-se que represente o custo de oportunidade de cessão de área da propriedade para a realização das atividades, deste modo, com uma correlação negativa nas probabilidades de participação e aceitação.

O fator Rentabilidade Pecuária apresenta variáveis produtivas e econômicas, tais como, a área total da propriedade, o número de cabeças de gado per capita, a área do lote destinado à pastagem e a renda agrícola mensal do indivíduo. Uma vez que denota diretamente o custo de oportunidade de abrir mão da atividade pecuária para a realização de outras atividades, espera-se que apresente correlação negativa com a disposição a participar e, principalmente, com a disposição a aceitar o valor oferecido. Caso registre uma correlação positiva, pode ser um indicativo de um viés de regressividade.

O fator Serviço Ambiental reflete o conhecimento do indivíduo sobre programas de pagamento por serviços ambientais. Espera-se um comportamento semelhante ao do fator de Percepção Ecológica.

No fator relativo à Inércia, as variáveis de destaque são as que se referem à indisposição do indivíduo para o câmbio de suas atividades habituais, bem como a aversão do mesmo a um possível monitoramento. Logo, espera-se uma correlação negativa com ambas as probabilidades a serem estimadas.

11 Os resultados da análise fatorial realizada encontram-se no Anexo 1 deste trabalho. 
O fator de Intensidade de Capital na Pecuária possui como variáveis de maiores cargas fatoriais aquelas que possuem relação com investimentos realizados na atividade pecuária. A princípio, espera-se que apresente uma correlação negativa com a disposição a participar da atividade de regeneração. Contudo, se apresentar uma correlação positiva, poderá indicar um possível viés de seleção adversa.

O fator Desconfiança possui uma relação estreita com o fato de o indivíduo ter dúvidas com relação à efetiva ocorrência do programa proposto. Assim como no caso do fator relativo à Inércia, espera-se que apresente correlação negativa com as probabilidades de participar e aceitar o montante oferecido pelo programa.

Já o fator Importância da Assistência destaca apenas as variáveis de percepção que revelam a necessidade de assistência técnica e, portanto, deve ter uma correlação negativa.

O fator de Disponibilidade Florestal revela uma estrutura na qual as variáveis que apresentam as maiores cargas fatoriais estão relacionadas com a dimensão do lote florestal da propriedade. Para a atividade de conservação florestal, espera-se que apresente uma correlação positiva, em virtude de expressar uma inicial conformidade da propriedade com relação ao escopo da atividade. Já para as atividades de regeneração e conversão, é possível que registre uma correlação negativa com a probabilidade de participar e aceitar pagamentos, uma vez que a maior relevância da área florestal na composição da propriedade faz com que o custo de oportunidade de cessão de áreas pertencentes às demais atividades seja incrementado.

O fator Custos de Produção reflete uma dimensão dos custos produtivos aos quais o proprietário está sujeito. Quanto maior for este fator, menor será o custo de oportunidade de se realizar o câmbio de suas atividades atuais. Por isso, espera-se uma correlação positiva com a probabilidade de participação nas atividades e na probabilidade de aceitar os pagamentos oferecidos.

O fator de Rentabilidade Agrícola explicita uma relação entre variáveis relativas ao montante de área utilizada para o plantio. Assim como no caso da Rentabilidade Pecuária, espera-se que apresente uma correlação negativa, pois quanto maior este for, maior será o custo de oportunidade de participar do programa. Uma correlação positiva pode indicar a presença de um viés de regressividade.

A Renda Não-Agropecuária apresenta como variável de maior carga fatorial o percentual da área dedicada à silvicultura. Além disso, destacam-se também as variáveis que versam sobre a renda que o indivíduo recebeu que não está ligada à atividade agrícola de sua propriedade ${ }^{12}$. Sua correlação positiva pode ser também um indicativo da ocorrência de um viés de regressividade.

12 Denominada de renda off-farm. 
Já o fator de Disponibilidade de Mão de Obra é caracterizado pela alta carga da variável que expressa o número de moradores na propriedade. Quanto maior for este fator, menor será o custo de oportunidade de realizar novas atividades; portanto, espera-se uma correlação positiva.

O fator Intensidade de Capital na Roça exibe como variáveis de maior carga fatorial aquelas que estão ligadas a investimentos realizados na atividade de roça. Espera-se um comportamento análogo ao do de Intensidade de Capital na Pecuária.

A Intensidade de Capital na Silvicultura aparece como o único fator em que a área plantada de eucalipto se destaca, refletindo o peso de tal atividade para o indivíduo. Quanto maior for a relevância deste fator para o entrevistado, maior será o custo de oportunidade para a implantação das atividades, em especial a de conservação. Portanto, espera-se uma correlação negativa.

O fator de Degradação Florestal reflete a condição de desmatamento vigente na propriedade. Quanto maior for a área degradada, menor será o custo de oportunidade para participação na atividade de regeneração e de conversão, podendo-se esperar uma correlação positiva com estes serviços.

O último fator retido na análise remonta à Capacidade de Financiamento do proprietário entrevistado. Quanto mais relevante for este fator para o indivíduo, menores tendem a ser os entraves para a participação no programa, podendo-se esperar uma correlação positiva com as atividades oferecidas.

\section{CONDICIONANTES DA PARTICIPAÇÃO}

Nossas estimativas com dois estágios apresentadas no Anexo 2 revelaram que para todas as atividades, a razão inversa de Mills não se mostrou estatisticamente significativa, indicando que o viés de seleção amostral não influiu de maneira representativa nas estimações realizadas para as funções de aceitação.

Com base nesses resultados, é possível afirmar que as estimativas das funções de participação e aceitação de pagamentos podem ser realizadas de maneira independente para cada uma das atividades oferecidas.

A Tabela 1 apresenta os condicionantes obtidos para as funções de participação no programa de PSA oferecido e para cada atividade proposta pelo mesmo. Como os entrevistados em geral respondem sua disposição a participar inicialmente no programa como um todo e depois em cada atividade, os resultados são estimados para cada uma dessas respostas.

Observa-se, de modo geral, que a participação no programa e em cada atividade é influenciada positivamente pelo nível de conhecimento prévio que o indivíduo possui sobre questões relacionadas à conservação ambiental e aos programas de PSA. Apenas para a atividade de conversão produtiva tais condicionantes não são significativos. 
Tabela 1 - Condicionantes da participação no programa e atividades

\begin{tabular}{|c|c|c|c|c|c|c|c|c|c|c|c|c|}
\hline \multirow{2}{*}{$\begin{array}{l}\begin{array}{c}\text { Participação no } \\
\text { Programa e } \\
\text { Serviços }\end{array} \\
\text { Variáveis }\end{array}$} & \multicolumn{3}{|c|}{ Todo Programa } & \multicolumn{3}{|c|}{$\begin{array}{c}\text { Na conservação } \\
\text { florestal }\end{array}$} & \multicolumn{3}{|c|}{$\begin{array}{l}\text { Na regeneração } \\
\text { florestal }\end{array}$} & \multicolumn{3}{|c|}{$\begin{array}{c}\text { Na conversão } \\
\text { produtiva }\end{array}$} \\
\hline & Coef. & $\begin{array}{c}\text { Erro } \\
\text { Padrão }\end{array}$ & $\mathrm{P}>|\mathrm{z}|$ & Coef. & $\begin{array}{c}\text { Erro } \\
\text { Padrão }\end{array}$ & $\mathrm{P}>|\mathrm{z}|$ & Coef. & $\begin{array}{c}\text { Erro } \\
\text { Padrão }\end{array}$ & $\mathrm{P}>|\mathrm{z}|$ & Coef. & $\begin{array}{c}\text { Erro } \\
\text { Padrão }\end{array}$ & $\mathrm{P}>|\mathrm{z}|$ \\
\hline Percepção Ecológica & 0,067 & 0,014 & 0,000 & 0,058 & 0,022 & 0,008 & 0,064 & 0,021 & 0,002 & - & - & - \\
\hline Inércia & $-0,087$ & 0,014 & 0,000 & $-0,086$ & 0,025 & 0,000 & $-0,055$ & 0,025 & 0,026 & $-0,096$ & 0,026 & 0,000 \\
\hline $\begin{array}{l}\text { Importância da } \\
\text { Assistência }\end{array}$ & 0,041 & 0,013 & 0,001 & - & - & - & - & - & - & - & - & - \\
\hline $\begin{array}{l}\text { Disponibilidade de } \\
\text { Mão de Obra }\end{array}$ & 0,073 & 0,017 & 0,000 & - & - & - & - & - & - & 0,078 & 0,023 & 0,001 \\
\hline $\begin{array}{l}\text { Intensidade de Capital } \\
\text { na Silvicultura }\end{array}$ & 0,060 & 0,026 & 0,022 & - & - & - & - & - & - & - & - & - \\
\hline MG & 0,056 & 0,029 & 0,052 & - & - & - & $-0,223$ & 0,052 & 0,000 & 0,233 & 0,049 & 0,000 \\
\hline $\begin{array}{l}\text { Entendeu o programa } \\
\text { de PSA }\end{array}$ & 0,100 & 0,034 & 0,003 & - & - & - & - & - & - & - & - & - \\
\hline Área Utilizada & - & - & - & $-0,027$ & 0,015 & 0,079 & - & - & - & - & - & - \\
\hline Custos & - & - & - & 0,047 & 0,025 & 0,057 & - & - & - & - & - & - \\
\hline $\begin{array}{l}\text { Renda Não- } \\
\text { Agropecuária }\end{array}$ & - & - & - & 0,052 & 0,026 & 0,042 & - & - & - & - & - & - \\
\hline $\begin{array}{l}\text { Participação em } \\
\text { Regeneração }\end{array}$ & - & - & - & $-0,080$ & 0,048 & 0,095 & - & - & - & 0,083 & 0,048 & 0,085 \\
\hline $\begin{array}{l}\text { Participação em } \\
\text { Conversão }\end{array}$ & - & - & - & $-0,202$ & 0,042 & 0,000 & 0,104 & 0,041 & 0,012 & - & - & - \\
\hline Questionário X & - & - & - & 0,131 & 0,042 & 0,002 & 0,077 & 0,041 & 0,059 & $-0,114$ & 0,044 & 0,010 \\
\hline RJ & - & - & - & - & - & - & $-0,170$ & 0,055 & 0,002 & 0,153 & 0,054 & 0,005 \\
\hline $\begin{array}{l}\text { Intensidade de Capital } \\
\text { na Roça }\end{array}$ & - & - & - & - & - & - & - & - & - & 0,071 & 0,026 & 0,006 \\
\hline Degradação Florestal & - & - & - & - & - & - & - & - & - & 0,063 & 0,024 & 0,008 \\
\hline $\begin{array}{l}\text { Participação em } \\
\text { Conservação }\end{array}$ & - & - & - & - & - & - & - & - & - & $-0,217$ & 0,050 & 0,000 \\
\hline $\begin{array}{l}\text { Número de } \\
\text { observações }\end{array}$ & 695,000 & - & - & 553,000 & - & - & 553,000 & - & - & 553,000 & - & - \\
\hline
\end{tabular}

Fonte: Elaboração própria. 
A importância da assistência técnica se mostra significativa apenas para aceitar a participação no programa de PSA como um todo.

Os fatores relativos à renda do indivíduo, quando têm significância na definição de sua participação sempre despontam com sinal positivo, indicando que quanto maior for a renda do indivíduo, maior será sua propensão a participar do esquema proposto. Este tipo de comportamento é um forte indicativo da presença de um viés de regressividade e somente não é registrado para a função de participação da atividade de regeneração florestal.

Como esperado, o sinal positivo oriundo do fator de disponibilidade florestal, isto é, menor a restrição de terra para cultivo, indica que quanto maior for a área de floresta na propriedade maior será a propensão do indivíduo em aceitar participar do esquema de PSA que lhe foi oferecido. Para o caso da atividade de conservação, a maior proporção de área de floresta expressa um menor custo de oportunidade do proprietário para a adoção de tal prática. Já para o caso da atividade de conversão produtiva, o fator de disponibilidade florestal registra um sinal positivo pelo fato de sua estrutura apresentar o pouco uso da área de floresta como a variável de maior carga fatorial. Uma vez que esta variável expressa uma atividade de conversão já iniciada, o sinal positivo apresentado pelo fator de disponibilidade florestal pode apontar em direção a um possível viés de seleção adversa.

No caso da atividade de regeneração florestal, o sinal negativo registrado pelo fator de disponibilidade florestal era esperado, uma vez que quanto maior a importância relativa da área de floresta na propriedade, maior o custo de cessão de novas áreas para este fim.

O fator que expressa a maior disponibilidade de mão de obra mostrou-se relevante e positivo apenas para a participação no programa e para a participação na atividade de conversão produtiva. $\mathrm{O}$ sinal positivo registrado em ambas as funções deriva do fato de que a maior disponibilidade de mão de obra diminui o custo de oportunidade de se realizar as atividades, em virtude do seu menor custo relativo para a realização da atividade.

O fator relativo à inércia dos proprietários para alterar suas atividades habituais registra uma influência negativa em todas as atividades oferecidas e também na participação no programa como um todo. Este resultado confirma a aversão dos produtores a novas técnicas de manejo e uso do solo.

Com exceção da função de participação em conservação, cabe ressaltar ainda as cargas positivas apresentadas pelas dummies indicativas de estado, indicando que as probabilidades de participação no programa variam regionalmente. Observa-se também que a participação em outra atividade afeta a decisão de participação na atividade oferecida ao usuário, com uma aparente economia de escopo sendo registrada com relação à execução conjunta das atividades de regeneração e conversão produtiva. 
Para a atividade de conservação, o modelo registra com sinal positivo a intensidade de capital que o indivíduo destina à atividade de pecuária. Este sinal era esperado, uma vez que, este fator representa o investimento feito em tecnologias poupadoras de terra, que abrem a possibilidade da expansão da atividade pecuária com um menor montante de terra, denotando um possível viés de seleção adversa.

Apenas a variável que mede a descrença do indivíduo na efetiva realização do programa apresentou um sinal divergente das expectativas a priori para a atividade de conservação, pois deveria representar um efeito negativo para a participação do mesmo. Nas outras atividades ela não apareceu significativa. De modo geral, observa-se, como esperado, que, quanto mais afeito é o indivíduo a questões relativas ao meio ambiente, maiores são as chances de participação na atividade de conservação.

Há também evidência de viés de regressividade quando a participação é positivamente condicionada à rentabilidade agrícola e à disponibilidade de mão de obra. Por fim, registra-se que a ordem de apresentação das atividades é relevante para a decisão do indivíduo, bem como o fato de já ter escolhido participar de outras atividades. Ou seja, quando um conjunto de atividades é apresentado a sequência de apresentação afeta o grau de participação.

Para a atividade de conversão produtiva, estimou-se que os fatores que medem o volume de investimentos concentrados na atividade de roça e na pecuária apresentam influência positiva na decisão de participar da atividade. Isto parece revelar um possível viés de seleção adversa, uma vez que indica uma maior probabilidade de participação para indivíduos que já implementam, em algum grau, a atividade proposta, comprometendo a adicionalidade do programa. Observa-se na Tabela 1 que, na participação no programa como um todo, o fator que mede a importância dada pelo entrevistado à prestação de assistência técnica e financeira a ser oferecida apresenta sinal positivo e, portanto, indicando que esse tipo de assistência é importante para a participação.

Como as ofertas de atividades de serviços ambientais propostas na pesquisa podem ser tanto financeira como tecnologicamente não triviais para muitos respondentes, analisamos a propensão a participar entre os usuários de acordo com seu nível de entendimento. A Tabela 2 apresenta os resultados do modelo Probit para a estimação dos condicionantes da participação no programa apenas para os indivíduos que declararam ter entendido bem o que a pesquisa estava propondo, considerando as três regiões em conjunto. 
Tabela 2 - Condicionantes da participação no programa dos que indicaram ter entendido o programa de PSA

\begin{tabular}{lccc}
\hline Variáveis & Coef. & Erro Padrão & $\mathbf{P}>|\mathbf{z}|$ \\
\hline Inércia & $-0,048$ & 0,013 & 0,000 \\
Disponibilidade Florestal & 0,041 & 0,011 & 0,000 \\
Rentabilidade Agrícola & 0,014 & 0,007 & 0,052 \\
Disponibilidade de Mão de & 0,042 & 0,012 & 0,001 \\
Obra & & & 0,001 \\
Intensidade de Capital na & 0,037 & 0,011 & 0,031 \\
Roça & 0,040 & & 0,002 \\
Intensidade de Capital na & 0,069 & 0,018 & 0,016 \\
Silvicultura & 0,054 & 0,023 & \\
RJ & 429,000 & 0,023 & \\
MG & 0,273 & & \\
Número de observações & $-113,688$ & & \\
Pseudo R2 & 73,990 & & \\
Log-Likelihood & 0,000 & & \\
Wald chi2(10) & & & \\
Prob > chi2 & & & \\
\hline
\end{tabular}

Fonte: Elaboração própria.

Como mostra a Tabela 2, os resultados são similares: quando o maior entendimento do programa leva o indivíduo a considerar fatores relativos ao nível de esforço que terá que ser empreendido para a adequação ao programa. Observa-se, assim, na Tabela 2 que aparecem, significativos e com os sinais esperados, os fatores que representam dificuldade em mudar suas atuais atividades (Inércia), a disponibilidade de recursos para a realização das atividades (Disponibilidade de Mão de Obra e de Disponibilidade Florestal) e qual o grau atual de implementação das atividades propostas (Intensidade de Capital na Roça). Ou seja, uma indicação clara de resistência a padrões tecnológicos alternativos associada à rentabilidade e nível de inversões do padrão atual. 


\section{CONDICIONANTES DA ACEITAÇÃO}

Os resultados da propensão a aceitar o pagamento oferecido são apresentados na Tabela 3.

Tabela 3 - Condicionantes da aceitação do pagamento

\begin{tabular}{|c|c|c|c|c|c|c|c|c|c|}
\hline \multirow{2}{*}{$\begin{array}{l}\begin{array}{c}\text { Aceitação do } \\
\text { Pagamento }\end{array} \\
\text { Variáveis }\end{array}$} & \multicolumn{3}{|c|}{ Na conservação florestal } & \multicolumn{3}{|c|}{ Na regeneração florestal } & \multicolumn{3}{|c|}{ Na conversão produtiva } \\
\hline & Coef. & $\begin{array}{c}\text { Erro } \\
\text { Padrão }\end{array}$ & $\mathrm{P}>|\mathrm{z}|$ & Coef. & $\begin{array}{c}\text { Erro } \\
\text { Padrão }\end{array}$ & $\mathrm{P}>|\mathrm{z}|$ & Coef. & $\begin{array}{c}\text { Erro } \\
\text { Padrão }\end{array}$ & $\mathrm{P}>|\mathrm{z}|$ \\
\hline $\begin{array}{l}\text { Valor de } \\
\text { pagamento para } \\
\text { conservação }\end{array}$ & 0,000 & 0,000 & 0,000 & - & - & - & - & - & - \\
\hline Área Utilizada & 0,713 & 0,263 & 0,007 & $-0,463$ & 0,171 & 0,007 & - & - & - \\
\hline $\begin{array}{l}\text { Disponibilidade } \\
\text { Florestal }\end{array}$ & 0,039 & 0,022 & 0,073 & 0,093 & 0,034 & 0,006 & - & - & - \\
\hline $\begin{array}{l}\text { Rentabilidade } \\
\text { Agrícola }\end{array}$ & $-0,055$ & 0,016 & 0,000 & - & - & - & - & - & - \\
\hline $\begin{array}{l}\text { Disponibilidade } \\
\text { de Mão de Obra }\end{array}$ & 0,061 & 0,019 & 0,001 & - & - & - & 0,053 & 0,022 & 0,015 \\
\hline $\begin{array}{l}\text { Participação em } \\
\text { Regeneração }\end{array}$ & 0,066 & 0,035 & 0,050 & - & - & - & - & - & - \\
\hline $\begin{array}{l}\text { Intensidade de } \\
\text { Capital na Roça }\end{array}$ & - & - & - & $-0,075$ & 0,031 & 0,017 & - & - & - \\
\hline $\begin{array}{l}\text { Valor de } \\
\text { pagamento para } \\
\text { conversão }\end{array}$ & - & - & - & - & - & - & 0,000 & 0,000 & 0,000 \\
\hline $\begin{array}{l}\text { Rentabilidade } \\
\text { Pecuária }\end{array}$ & - & - & - & - & - & - & $-0,038$ & 0,019 & 0,041 \\
\hline Desconfiança & - & - & - & - & - & - & $-0,035$ & 0,020 & 0,078 \\
\hline Questionário X & - & - & - & - & - & - & 0,077 & 0,040 & 0,051 \\
\hline $\begin{array}{l}\text { Número de } \\
\text { observações }\end{array}$ & 341,000 & - & - & 178,000 & - & - & 320,000 & - & - \\
\hline Pseudo R2 & 0,166 & - & - & 0,078 & - & - & 0,118 & - & - \\
\hline Log-Likelihood & $-133,541$ & - & - & $-90,696$ & - & - & $-132,225$ & - & - \\
\hline Wald chi2(10) & 41,400 & - & - & 16,710 & - & - & 30,040 & - & - \\
\hline Prob $>$ chi 2 & 0,000 & - & - & 0,001 & - & - & 0,000 & - & - \\
\hline
\end{tabular}

Fonte: Elaboração própria.

Observa-se na Tabela 3 que há uma correlação positiva com o valor oferecido ao indivíduo para as atividades de conservação e conversão produtiva, estando de acordo com o previsto na literatura. Contudo, para o caso da atividade de regeneração, isto não ocorreu. Esta ausência de significância estatística deve-se, possivelmente, à baixa participação neste serviço, que comprometeu o tamanho da amostra.

Nota-se que o fator relativo à disponibilidade de mão de obra apresenta uma influência positiva na aceitação do valor oferecido, refletindo a ideia de que um menor 
custo de mão de obra aumenta as chances de aceitação do pagamento na medida em que evidencia um menor custo de oportunidade.

Ao contrário do ocorrido para a participação na atividade de conservação, nesse caso, desponta como estatisticamente significativo e com sinal positivo o fator relativo à dimensão dos lotes utilizados na propriedade, sendo possivelmente explicada pela alta carga fatorial da dimensão da área de floresta na estrutura deste fator, indicando um menor custo de oportunidade para a implementação da atividade. Já para a atividade de regeneração florestal, este fator apresenta sinal negativo, indicando que, quanto maior for a área utilizada na propriedade, maior será o custo para converter esta terra em área de mata nativa. Como esperado, este fator não exerce influência para a aceitação da atividade de conversão produtiva.

A Tabela 3 mostra que a renda agrícola está negativamente correlacionada à aceitação do valor oferecido para participação no serviço de conservação florestal, diferentemente da decisão de participar na mesma atividade. Já a renda pecuária está negativamente correlacionada à decisão de aceitar o valor oferecido para a realização da atividade de conversão produtiva, sendo contrário ao sinal encontrado na função de participação da atividade de conversão produtiva. Com relação à atividade de regeneração produtiva, a baixa participação na atividade parece ter exercido influência na estimação da função de aceitação, com o registro de alguns resultados anômalos. São os casos do sinal positivo do fator de disponibilidade florestal e a ausência de significância estatística da variável relativa ao valor oferecido pela realização da atividade.

Por fim, a atividade de conversão produtiva apresenta algumas peculiaridades em relação às demais atividades. A primeira delas diz respeito à presença do fator de desconfiança como significativo na decisão de aceitar pagamentos. O coeficiente negativo deste fator parece indicar que quanto maior a incerteza com relação ao bom funcionamento do programa, menor é a probabilidade de se aceitar o valor oferecido por ele, exigindo-se uma remuneração maior. A explicação para a presença deste fator pode ser extraída do receio dos proprietários em mudar suas atividades habituais de produção para atenderem às demandas do programa, tal qual reflete o fator de inércia, registrado na função de participação deste serviço.

Por último, o coeficiente positivo obtido para a variável de ordenamento das atividades no questionário indica que quando a atividade de conversão é apresentada por último, aumenta a probabilidade de aceitação do pagamento oferecido por esta atividade, diferentemente do resultado encontrado no modelo de participação para a mesma atividade. Isto sugere a possibilidade de um efeito de sequência.

Em suma, na condicionalidade da aceitação do pagamento dominam os fatores relacionados com o custo de oportunidade do esforço de provisão e muito menos os de percepção que, como analisado anteriormente, foram importantes para aceitar participar no programa. 


\section{DISCUSSÃO E CONCLUSÃO}

Neste estudo, foram estimadas as disposições a participar e a aceitar pagamentos para um programa de pagamentos por serviços ambientais na região do bioma da Mata Atlântica. Para tal, foram analisados os condicionantes de tais decisões, com base em fatores relativos às características econômicas, produtivas e também com relação às preferências dos indivíduos entrevistados, partindo-se da premissa inicial da existência de endogeneidade entre as funções de participação e aceitação de cada atividade. Entretanto, nossos resultados demonstram que não há o viés significativo de seleção amostral e, portanto, a estimativa das funções em separado continua sendo a mais adequada.

Além de estarem alinhados com as principais evidências na literatura internacional ao detectarem que a participação em programas de PSA é afetada pelo custo de oportunidade com viés de regressividade, aversão a risco e restrição de crédito, nossos resultados são muito similares aos encontrados por Seroa da Motta e Ortiz (2018) que utilizaram a mesma base com estratégia de identificação distinta. Mas, como os nossos indicadores são baseados em análise fatorial, acreditamos ter contribuído para melhorar a interpretação das variáveis condicionantes.

Nossos resultados apontam que o maior nível de entendimento dos entrevistados acerca de questões relativas à conservação ambiental e também aos programas de PSA exerce uma influência positiva na probabilidade de adesão, tanto ao programa quanto as atividades, individualmente.

Por outro lado, há uma indicação muito forte de que o receio dos proprietários rurais em relação aos riscos de adesão ao programa em termos de sua continuidade e excesso de monitoramento como também na incerteza sobre atividades ofertada, reduzem a disposição em participar do mesmo. Portanto, reitera-se a necessidade de instituição de ações que visem aumentar o nível de informação disponível sobre o programa de PSA e seus benefícios para a preservação ambiental como forma de potencializar sua taxa de adesão.

Como também apontado na literatura, o efeito da renda nas decisões dos indivíduos é, na maioria dos casos, positivo para a participação, indicando a presença de um possível viés de regressividade. Contudo, para as funções de aceitação de pagamentos, os fatores de renda apresentaram correlações negativas, denotando uma relação expressa com o custo de oportunidade de se abrir mão de atividades atuais (geradoras de renda) em prol das atividades propostas no programa, um resultado que não aparecia tão claramente em Seroa da Motta e Ortiz (2018).

Confirmamos também que a adequação da propriedade às atividades propostas, dada pela correlação positiva e estatisticamente significativa aos investimentos já rea- 
lizados (intensidade de capital), desponta como um importante fator na decisão do entrevistado em participar do programa. O que representa uma tendência a atrair os indivíduos que já implementam as atividades propostas, ou seja, um viés de seleção adversa que pode comprometer a hipótese de adicionalidade do programa.

O fato de a dummy relativa ao tipo de questionário utilizado ser estatisticamente significativa nos modelos de participação e de aceitação das atividades indica que a ordem de apresentação destas é relevante na probabilidade de participar das mesmas, o que não aparece em Seroa da Motta e Ortiz (2018). Ou seja, no desenho do programa há que se considerar que o nível de adesão é sensível à sequência de oferta destes.

Adicionalmente, confirmamos que quando um serviço é oferecido antes dos demais, aumenta a propensão da participação no mesmo, indicando, uma possível economia de escopo. Assim, registra-se a presença de sinergias em relação à adesão das atividades e também com relação à probabilidade de aceitação do pagamento oferecido. É explicitada uma possível relação de complementariedade entre as atividades de regeneração e conversão produtiva. Além disso, registra-se que o fato de participar de outra atividade aumenta as chances de aceitar o pagamento oferecido, ao menos no caso da atividade de conservação.

Confirma-se também a hipótese de que as características regionais influenciam na decisão de participar do programa e, portanto, o sucesso do programa pode estar associado a sua cobertura geográfica.

Em suma, nossos resultados confirmam de forma muito evidente que, além de fatores financeiros e produtivos, os condicionantes de percepção relativos a desconfiança e inércia dos produtores afetam negativamente a adesão em programas de PSA e que há um forte viés de seleção adversa. Portanto, no desenho desses programas se faz necessário criar mecanismos no sentido de reduzir o grau de incerteza sobre as atividades de provisão a serem empreendidas e da própria gestão do programa.

\section{REFERÊNCIAS}

ALBERINI, A.; KAHN, J. (Eds.). Handbook on contingent valuation. Cheltenham, U.K.: Edward Elgar Publishing, 2006.

ALPIZAR, F.; BLACKMAN, A.; PFAFF, A. Payments for ecosystem services: why precision and targeting matter. Resources, v. 165, p. 20-22, 2007.

ARROW, K. et al. Report of the NOAA Panel on contingent valuation. Federal Register, v. 58, n. 10, p. 4601-4614, 1993.

AMIGUES, J. et al. The benefits and costs of riparian analysis habitat preservation: a willingness to accept/willingness to pay contingent valuation approach. Ecological Economics, v. 43, n. 1, p. 17-31, 2002. 
BATEMAN, I. J.; LANGFORD, I. H. Budget constraint, temporal, and question-ordering effects in contingent valuation studies. Environment and Planning A, v. 28, p. 1215-1228, 1997.

BATEMAN, I. J. et al. Economic valuation with stated preference techniques: a manual. Cheltenham, U.K.: Edward Elgar Publishing, 2002.

BAUMOL, W.; OATES, W. The theory of environmental policy. 2. ed. Cambridge, UK.: Cambridge University Press, 1990.

BROCH, S. W.; VEDEL, S. E. Using choice experiments to investigate the policy relevance of heterogeneity in farmer agri-environmental contract preferences. Environmental and Resource Economics, v. 51, n. 4, p. 561-581, 2012.

BUCKLEY, C.; HYNES, S.; MECHAN, S. Supply of an ecosystem service-farmers' willingness to adopt riparian buffer zones in agricultural catchments. Environmental Science \& Policy, v. 24, n. 1, p. 101-109, 2012.

BUSH, G. et al. Measuring the local costs of conservation: a provision point mechanism for eliciting willingness to accept compensation. Land Economics, v. 89, n. 3, p. 490-513, 2013.

FAO - FOOD AND AGRICULTURE ORGANIZATION. Payments for ecosystem services and food security. Roma: FAO, 2011.

CRANFORD, M.; MOURATO, S. Community conservation and a two-stage approach to payments for ecosystem services. Ecological Economics, v. 71, p. 89-98, 2011.

ESPINOSA, M.; BARREIRO-HURLÉ, J.; RUTO, R. What do farmers want from agri-environmental scheme design? A choice experiment approach. Journal of Agricultural Economics, v. 61, n. 2, p. 259-273, 2010.

ENGEL, S.; PAGIOLA, S.; WUNDER, S. Designing payments for environmental services in theory and practice: An overview of the issues. Ecological Economics, v. 65, n. 4, p. 663-674, 2008.

FERRARO, P. J.; KISS, A. Direct payments to conserve biodiversity. Science, v. 298, n. 5599, p. 1718-1719, 2002.

GUEDES, F. B.; SEEHUSEN, S. Pagamento por serviços ambientais na Mata Atlântica: Lições aprendidas e desafios. Brasília, D. F.: Ministério do Meio Ambiente, 2011.

GRAMMATIKOPOULOUA, I.; IHOA, A.; POUTA, E. Willingness of farmers to participate in agri-environmental auctions in Finland. Food Economics, v. 9, n. 4, p. 215-230, 2013.

GOMEZ-BAGGETHUN, E. et al. The history of ecosystem services in economic theory and practice: from early notions to markets and payment schemes. Ecological Economics, v. 69, p. 209-1218, 2010.

GROSJEAN, P.; KONTOLEON, A. How sustainable are sustainable development programs? The case of the sloping land conversion program in China. World Development, v. 37, n. 1, p. 268-285, 2009.

HAIR, J. F. et al. Multivariate data analysis. 7. ed. [S.l.]: Prentice Hall, feb. 2009.

HECKMAN, J. J. Sample selection bias as a specification error. Econometrica, v. 47, n. 11, p. 153 $161,1979$. 
LAVRATTI, P.; TEJEIRO, G. (Orgs.). Pagamento por serviços ambientais: experiências locais e latino-americanas. Direito e Mudanças Climáticas, Instituto O Direito por um Planeta Verde, v. 7, 2014.

KILGORE, M. A. et al. What does it take to get family forest owners to enroll in a forest stewardship-type program? Forest Policy and Economics, v. 10, n. 7-8, p. 507-514, 2008.

KERR, J. M. et al. Long-term effects of payments for environmental services: combining insights from Communication and Economics. Sustainability, v. 9, n. 9, p. 3-13, 2017.

KLINE, J. D.; ALIG, R. J.; JOHNSON, R. L. Forest owner incentives to protect riparian habitat. Ecological Economics, v. 33, n. 1, p. 29-43, 2000.

LEIMONA, B. ET. AL. Boundary work: Knowledge co-production for negotiating payment for watershed services in Indonesia. Ecosystem Services, v. 15, p. 45-62, 2015.

LINDHJEMA, H.; MITANI, Y. Forest owners' willingness to accept compensation for voluntary conservation: a contingent valuation approach. Journal of Forest Economics, v. 18, n. 4, p. 290-302, 2012.

MARTÍNEZ-CRUZ, D. A. et al. Disposición de los produtores forestales de la región izta-popo a aceptar pagos por mantener los servicios ambientales hidrológicos. Tropical and Subtropical Agroecosystems, v. 12, n. 3, p. 549-556, 2010.

MARTIN-ORTEGA, J.; OJEA, E.; CAMILLE ROUX, C. Payments for water ecosystem services in Latin America: a literature review and conceptual model. Ecosystem Services, v. 6, p. 122-132, 2013.

MAY, P. H. et al. Assessment of the role of economic and regulatory instruments in the conservation policymix for the Brazilian Amazon - a coarse grain analysis. Policy Mix, v. 5, 2012.

MITCHELL, R. C.; CARSON, R. T. Using surveys to value public goods: the contingent valuation method. Washington, D.C.: RFF Press, 1989.

MURADIAN, R. et al. Reconciling theory and practice: an alternative conceptual framework for understanding payments for environmental services. Ecological Economics, v. 69, n. 6, p. 1202-1208, 2010.

OECD - ORGANISATION FOR ECONOMIC CO-OPERATION AND DEVELOPMENT. Paying for biodiversity: enhancing the cost-effectiveness of payments for ecosystems services. Paris: OECD, 2010.

PAGIOLA, S.; LANDELL-MILLS, N.; BISHOP, J. "Making market-based mechanisms work for forests and people”. In: PAGIOLA, S.; BISHOP, J.; LANDELL-MILLS, N. (Eds.). Selling forest environmental services: market-based mechanisms for conservation and development. London: Earthscan, 2002.

PAGIOLA, S. Assessing the efficiency of payments for environmental services programs: a framework for analysis. Washington, D.C.: World Bank, 2005.

PAGIOLA, S.; PLATAIS, G. Payments for environmental services: from theory to practice. Washington, D. C.: World Bank, 2007.

PESCHE, D. et al. "Ecosystem services: emergence of new paradigms within the economics of ecosystem”. In: MURADIAN, R.; RIVAL, L. (Eds.). Governing the provision of environmental services. Springer, 2012. 
POE, G. L. et al. Provision point mechanisms and field validity tests of contingent valuation. Environmental and Resource Economics, v. 23, n. 1, p. 105-131, 2002.

RUTO, E. S. K.; GARROD, G. D. Investigating farmers' preferences for the design of agri-environment schemes: a choice experiment approach. Journal of Environmental Planning and Management, v. 52, n. 9, p. 631-647, 2009.

SEROA DA MOTTA, R. Economia ambiental. Rio de Janeiro: Editora FGV, 2006.

SEROA DA MOTTA; ORTIZ, R. A. Costs and perceptions conditioning willingness to accept payments for ecosystem services in a Brazilian case. Ecological Economics, v. 147, p. 333$342,2018$.

SEROA DA MOTTA, R. Manual para valoração econômica de recursos ambientais. Rio de Janeiro e Brasília: IPEA/MMA/PNUD/CNPq, 1997.

SULLIVAN, J.; AMACHER, G. S.; CHAPMAN, S. Forest banking and forest landowners forgoing management rights for guaranteed financial returns. Forest Policy and Economics, v. 7, n. 3, p. 381-392, 2005.

VANSLEMBROUCK, I.; VAN HUYLENBROECK, G.; VERBEKE, W. Determinants of the willingness of Belgian farmers to participate in agri-environmental measures. Journal of Agricultural Economics, v. 53, n. 3, p. 489-511, nov. 2002.

VATN, A. An institutional analysis of payments for environmental services. Ecological Economics, v. 69, p. 245-1252, 2010.

VIJESH, V. et al. Estimating compensation payments for on-farm conservation of agricultural biodiversity in developing countries. Ecological Economics, v. 87, p.110-123, 2013.

WEI, X.; GUAN, Z.; ZHU, H. Farmer's willingness to participate in wetland restoration: a hurdle model approach. Agricultural, Economics, v. 47, n. 6, p. 719-27, 2016.

WUNDER, S. Payments for environmental services and the poor: concepts and preliminary evidence. Environment and Development Economics, p. 13, n. 3, p. 79-97, 2008.

WUNDER, S.; ENGEL, S.; PAGIOLA, S. Taking stock: a comparative analysis of payments for environmental services programs in developed and developing countries. Ecological Economics, v. 65, n. 4, p. 834-852, 2008.

YOUNG, C. E. F.; BAKKER, L. B. D. "Instrumentos econômicos e pagamentos por serviços ambientais no Brasil”. In: FOREST TRENDS (Ed.) Incentivos Econômicos para Serviços Ecossistêmicos no Brasil. Rio de Janeiro: Forest Trends, 2015, p. 33-56.

ZANELLA, M. A.; SCHLEYER, C.; SPEELMAN, S. Why do farmers join Payments for Ecosystem Services (PES) schemes? An Assessment of PES water scheme participation in Brazil. Ecological Economics, v. 105; p. 166-176, 2014. 


\section{ANEXO 1 - RESULTADOS DA ANÁLISE FATORIAL}

A seguir são apresentados os resultados da análise fatorial com as características dos fatores considerados e as respectivas pontuaçõesfatoriais de cada variável em cada um deles.

Tabela A.1 - Resultados da análise fatorial

\begin{tabular}{|c|c|c|c|c|}
\hline FATOR & AUTOVALOR & DIFERENÇA & PROPORÇÃO & CUMULATIVO \\
\hline Percepção Ecológica & 5,186 & 1,214 & 0,098 & 0,098 \\
\hline Área Utilizada & 3,973 & 0,664 & 0,075 & 0,173 \\
\hline Rentabilidade Pecuária & 3,309 & 0,697 & 0,062 & 0,235 \\
\hline Serviço Ambiental & 2,612 & 0,282 & 0,049 & 0,285 \\
\hline Inércia & 2,330 & 0,504 & 0,044 & 0,329 \\
\hline $\begin{array}{l}\text { Intensidade de Capital na } \\
\text { Pecuária }\end{array}$ & 1,826 & 0,138 & 0,035 & 0,363 \\
\hline Desconfiança & 1,688 & 0,232 & 0,032 & 0,395 \\
\hline Importância da Assistência & 1,455 & 0,021 & 0,028 & 0,422 \\
\hline Disponibilidade Florestal & 1,434 & 0,069 & 0,027 & 0,449 \\
\hline Custos de Produção & 1,366 & 0,083 & 0,026 & 0,475 \\
\hline Rentabilidade Agrícola & 1,282 & 0,036 & 0,024 & 0,499 \\
\hline Renda Não-Agropecuária & 1,246 & 0,077 & 0,024 & 0,523 \\
\hline Pequeno Porte & 1,169 & 0,048 & 0,022 & 0,545 \\
\hline $\begin{array}{l}\text { Intensidade de Capital na } \\
\text { Roça }\end{array}$ & 1,120 & 0,035 & 0,021 & 0,566 \\
\hline $\begin{array}{l}\text { Intensidade de Capital na } \\
\text { Silvicultura }\end{array}$ & 1,085 & 0,039 & 0,021 & 0,586 \\
\hline Degradação Florestal & 1,046 & 0,024 & 0,020 & 0,606 \\
\hline $\begin{array}{l}\text { Capacidade de } \\
\text { Financiamento }\end{array}$ & 1,022 & 0,023 & 0,019 & 0,626 \\
\hline
\end{tabular}

\section{ANEXO 2 - RESULTADOS DA ABORDAGEM EM DOIS ESTÁGIOS DE HECKMAN}

A seguir são apresentados os resultados para as funções de aceitação do pagamento oferecido, corrigidas segundo a metodologia em dois estágios de Heckman. Nota-se que em nenhuma das estimações a razão inversa de Mills foi significativa. 
Anexo 2 - Resultados da abordagem em dois estágios de Heckman

\begin{tabular}{|c|c|c|c|}
\hline \multicolumn{4}{|c|}{ Conservação } \\
\hline Variáveis & Coef. & Erro Padrão & $\mathbf{P}>|\mathbf{z}|$ \\
\hline $\begin{array}{l}\text { Valor de pagamento para } \\
\text { conservação }\end{array}$ & 0,000 & 0,000 & 0,000 \\
\hline Área Utilizada & 0,734 & 0,272 & 0,007 \\
\hline Serviço Ambiental & $-0,034$ & 0,020 & 0,083 \\
\hline Rentabilidade Agrícola & $-0,061$ & 0,016 & 0,000 \\
\hline Disponibilidade de Mão de Obra & 0,058 & 0,018 & 0,002 \\
\hline Participação em Conversão & 0,073 & 0,040 & 0,067 \\
\hline Razão Inversa de Mills & $-0,053$ & 0,063 & 0,401 \\
\hline Número de observações & 341,000 & & \\
\hline Pseudo R2 & 0,166 & & \\
\hline Log-Likelihood & $-133,641$ & & \\
\hline Wald chi2(7) & 43,080 & & \\
\hline Prob $>$ chi2 & 0,000 & & \\
\hline \multicolumn{4}{|c|}{ Regeneração } \\
\hline Área Utilizada & $-0,466$ & 0,172 & 0,007 \\
\hline Disponibilidade Florestal & 0,095 & 0,037 & 0,009 \\
\hline Intensidade de Capital na Roça & $-0,075$ & 0,031 & 0,016 \\
\hline Razão Inversa de Mills & $-0,018$ & 0,123 & 0,884 \\
\hline Número de observações & 178,000 & & \\
\hline Pseudo R2 & 0,079 & & \\
\hline Log-Likelihood & $-90,687$ & & \\
\hline Wald chi2(4) & 17,260 & & \\
\hline Prob $>$ chi2 & 0,002 & & \\
\hline \multicolumn{4}{|c|}{ Conversão } \\
\hline Valor de pagamento para conversão & 0,000 & 0,000 & 0,000 \\
\hline Rentabilidade Pecuária & $-0,039$ & 0,019 & 0,039 \\
\hline Desconfiança & $-0,035$ & 0,020 & 0,077 \\
\hline Disponibilidade de Mão de Obra & 0,051 & 0,023 & 0,025 \\
\hline Questionário X & 0,079 & 0,040 & 0,047 \\
\hline Razão Inversa de Mills & $-0,016$ & 0,103 & 0,876 \\
\hline Número de observações & 320,000 & & \\
\hline Pseudo R2 & 0,118 & & \\
\hline Log-Likelihood & $-132,206$ & & \\
\hline Wald chi2(6) & 30,380 & & \\
\hline Prob $>$ chi 2 & 0,000 & & \\
\hline
\end{tabular}

